\title{
Renormalizability of center-vortex sectors in continuum Yang-Mills theory
}

\author{
D. Fiorentini ${ }^{1}, D$. R. Junior ${ }^{1,2, *}, L$. E. Oxman ${ }^{1}$, and R. F. Sobreiro ${ }^{1}$ \\ ${ }^{1}$ Instituto de Física, Universidade Federal Fluminense, \\ Av. Litorânea s/n, 24210-346, Niterói, RJ, Brasil. \\ ${ }^{2}$ Institut für Theoretische Physik, Universität Tübingen, \\ Auf der Morgenstelle 14, 72076, Tübingen, Deutschland.
}

\begin{abstract}
Recently, a novel approach to quantize SU(N) Yang-Mills theory was proposed, where the configuration space $\left\{A_{\mu}\right\}$ is split into sectors labeled by topological defects, and then the gauge is fixed by a sector dependent condition. As the procedure is local in $\left\{A_{\mu}\right\}$, it could be free from Gribov copies. In this work, we review the renormalizability of sectors labeled by an arbitrary number of elementary center vortices.
\end{abstract}

Because of the Gribov problem, the understanding of continuum Yang-Mills theory in the infrared regime is certainly not within reach of the usual Fadeev-Popov method. This obstruction was established in Refs. [1,2], where it was shown that there is no continuous, global gauge fixing condition in configuration space $\left\{A_{\mu}\right\}$, when the gauge group is $S U(N)$. One possible approach to circumvent this problem is to restrict the path integral to the first Gribov region, which was followed in Refs. [3-9].

An alternative approach was proposed by Singer in his seminal paper, which relies on a locally finite open covering $\left\{\vartheta_{\alpha}\right\}$ of the space of all gauge fields $\left\{A_{\mu}\right\}$, with a subordinate partition of unity

$$
\sum_{\alpha} \rho_{\alpha}\left(A_{\mu}\right)=1 \quad, \quad \forall A_{\mu} \in\left\{A_{\mu}\right\}
$$

with the support of the function $\rho_{\alpha}\left(A_{\mu}\right)$ being on $\vartheta_{\alpha}$. The idea is to insert this identity in the Yang-Mills partition function, as follows

$$
Z_{Y M}=\sum_{\alpha} Z_{(\alpha)} \quad, \quad Z_{(\alpha)}=\int_{\vartheta_{\alpha}}[D A] \rho_{\alpha}(A) e^{-S_{Y M}(A)},
$$

where the sets $\left\{\vartheta_{\alpha}\right\}$ are to be chosen so as to guarantee the existence of local gauge-fixing conditions $f_{\alpha}(A)=0, A \in \vartheta_{\alpha}$, which are free from Gribov copies. Recently, a concrete implementation of this procedure was proposed for the case where the sets $\vartheta_{\alpha}$ are disjoint, thus forming a partition of $\left\{A_{\mu}\right\}[10]$. The first step is to introduce auxiliary adjoint scalar fields $\psi_{I}$ with an auxiliary action $S_{a u x}(\psi, A)$ in the theory by means of an identity. Each gauge field is then correlated with the $\zeta_{I}(A)$ that minimizes $S_{\text {aux }}(A, \zeta)$. The next step is to perform a generalized polar decomposition on the tuple $\zeta_{I}(A)$, which allow us to write $\zeta_{I}(A)=$ $S(A) q_{I}(A) S^{-1}(A), S \in S U(N)$. The tuple $q_{I}$ is the rotated $\zeta_{I}$ which is closest to a given

\footnotetext{
*Presenter; e-mail: davidjunior@id.uff.br.
} 
element of $\mathcal{M}$, the vacuum manifold of $S_{\text {aux }}$. We choose this element to be $v T_{I}$, so that $q_{I}$ satisfies the generalized pure modulus condition $\left[q_{I}, T_{I}\right]=0$. One important fact is that the phase $S$ will not always be regular. In general, we may write $S=U S_{0}$, with $U$ regular. We then introduce an equivalence class, where two phases are said to be equivalent if they differ by a regular gauge transformation, i.e.

$$
[S]=\left[S^{\prime}\right] \Longleftrightarrow S=U S^{\prime},
$$

for some regular $U$. Then, it is natural to split the configuration space $\left\{A_{\mu}\right\}$ into sectors $\mathcal{V}_{S_{0}}$, where $A_{\mu} \in \mathcal{V}_{S_{0}} \Longleftrightarrow[S]=\left[S_{0}\right]$. Because of the structure of $S_{\text {aux }}$, the labels $S_{0}$ are given by center vortex configurations. The gauge is then fixed by the sector dependent gauge condition

$$
f_{S_{0}}(\psi(A))=\left[\psi_{I}(A), S_{0} T_{I} S_{0}^{-1}\right]=0 .
$$

In this case, Eq. (2) becomes

$$
Z_{Y M}=\sum_{S_{0}} Z_{Y M}^{S_{0}} \quad, \quad Z_{Y M}^{S_{0}}=\left.\int_{\vartheta\left(S_{0}\right)}[D A] e^{-S_{Y M}} \delta\left(f_{S_{0}}(A)\right) \operatorname{Det} \frac{\delta f_{S_{0}}\left(A^{U}\right)}{\delta U}\right|_{U=I} .
$$

Observables may be similarly evaluated by a sum over the partial contributions of the sectors, i.e.

$$
\begin{aligned}
& \langle O\rangle=\sum_{S_{0}} \frac{Z_{Y M}^{S_{0}}}{Z_{Y M}}\langle O\rangle_{S_{0}}, \\
& \langle O\rangle_{S_{0}}=\left.\int_{\vartheta\left(S_{0}\right)}[D A] O(A) e^{-S_{Y M}} \delta\left(f_{S_{0}}(A)\right) \operatorname{Det} \frac{\delta \mathrm{f}_{\mathrm{S}_{0}}\left(\mathrm{~A}^{\mathrm{U}}\right)}{\delta \mathrm{U}}\right|_{\mathrm{U}=\mathrm{I}} .
\end{aligned}
$$

Eq. (6) resembles the type of expressions which are considered in phenomenological approaches involving center vortex ensembles (see the reviews [11, 12], and references therein). In these approaches, averages of the Wilson Loop are represented by a sum over center vortex configurations with a weight that is chosen on phenomenological grounds. Therefore, this quantization procedure not only has the potential to avoid the Gribov problem, but also provides a glimpse of a path from Yang-Mills theory to an ensemble of center vortices. An important line of investigation is then to compute the partial contribution of each vortex sector from first principles in order to derive and/or improve the phenomenological approach. A relevant first step in this direction is to establish the renormalizability of the partial contributions $Z_{S_{0}}$. This was firstly studied for the vortex-free sector in Ref. [13], and then generalized to a sector containing a general oriented elementary center-vortex configuration in Ref. [14].

In section 1 we present the sector-dependent gauge fixed action that implements the conditions of Eq. (4). In section 2 we review the renormalizability of the vortex-free sector. In section 3 we review the generalization of this result to a sector labeled by an arbitrary number of elementary oriented center vortices. In section 4 we present our conclusions.

\section{The gauge-fixed action}

From Eq. (5) it is clear that the action will be a sector dependent functional of the fields, as the gauge fixing condition depends on $\mathcal{V}\left(S_{0}\right)$. In order to obtain a concrete expression for it, we introduce in each $Z_{Y M}^{S_{0}}$ the identity

$$
1=\int\left[D \zeta_{I}\right] \delta\left(\frac{\delta S_{a u x}}{\delta \zeta_{I}}\right) \operatorname{det}\left(\frac{\delta^{2} S_{a u x}}{\delta \zeta_{I} \delta \zeta_{J}}\right)
$$


which correlates each gauge field $A_{\mu}$ with the tuple $\zeta_{I}(A)$ that minimizes $S_{a u x}$ in the presence of $A_{\mu}$. As discussed in Ref. [15], it is important to choose $S_{\text {aux }}$ carefully in order to obtain a well-defined mapping $A_{\mu} \rightarrow \zeta_{I}(A)$. In particular, this action should contain Spontaneous Symmetry Breaking (SSB) in order to avoid problems with the injectivity of the map. We considered the choice

$$
\begin{aligned}
S_{a u x}(\zeta, A)= & \int d^{4} x\left(D_{\mu}^{a b} \zeta_{I}^{b} D_{\mu}^{a c} \zeta_{I}^{c}+\mu^{2} \zeta_{I}^{a} \zeta_{I}^{a}+\kappa f_{I J K} f^{a b c} \zeta_{I}^{a} \zeta_{J}^{b} \zeta_{K}^{c}\right. \\
& \left.+\lambda \gamma_{I J K L}^{a b c d} \zeta_{I}^{a} \zeta_{J}^{b} \zeta_{K}^{c} \zeta_{L}^{d}\right),
\end{aligned}
$$

the tensor $\gamma$ being a general combination of structure constants and deltas. The delta functionals and determinants in Eq. (7) are exponentiated using flavored Lie algebra valued ghost $\left(\bar{c}_{I}, c_{I}\right)$ and Lagrange multiplier $\left(b_{I}\right)$ fields. Similarly, the gauge-fixing terms in Eq. (5) are exponentiated by $\bar{c}, c$ and $b$. The resulting additional action is given by

$$
\begin{aligned}
\Sigma\left(S_{0}\right)= & \int d^{4} x\left(D_{\mu}^{a b} b_{I}^{b} D_{\mu}^{a c} \zeta_{I}^{c}+D_{\mu}^{a b} \bar{c}_{I}^{b} D_{\mu}^{a c} c_{I}^{c}+\mu^{2}\left(\bar{c}_{I}^{a} c_{I}^{a}+b_{I}^{a} \zeta_{I}^{b}\right)+\right. \\
& \kappa f_{I J K} f^{a b c}\left(b_{I}^{a} \zeta_{J}^{b} \zeta_{K}^{c}-2 \bar{c}_{I}^{a} \zeta_{K}^{b} c_{J}^{c}\right)+\lambda \gamma_{I J K L}^{a b c d}\left(b_{I}^{a} \zeta_{J}^{b} \zeta_{K}^{c} \zeta_{L}^{d}+3 \bar{c}_{I}^{a} c_{J}^{b} \zeta_{K}^{c} \zeta_{L}^{d}\right) \\
& -U^{2} \bar{c}_{I}^{a} \zeta_{I}^{a}-\Lambda f^{a b c} f^{c d e} \bar{c}_{I}^{a} \zeta_{J}^{b} \zeta_{I}^{d} \zeta_{J}^{e}-\mathcal{K} f^{I J K} f^{a b c} \bar{c}_{I}^{a} \zeta_{J}^{b} \zeta_{K}^{c} \\
& \left.+i f^{a b c}\left(b^{a} \eta_{I}^{b} \zeta_{I}^{c}+\bar{c}^{a} \eta_{I}^{b} c_{I}^{c}\right)+f^{e c d} f^{e b a} \bar{c}^{a} \eta_{I}^{b} \zeta_{I}^{c} c^{d}\right)
\end{aligned}
$$

where the background field $\eta_{I}=v S_{0} T_{I} S_{0}^{-1}$ implements the sector-dependent condition of Eq. (4). It is possible to show that this action has a BRST symmetry, given by

$$
\begin{aligned}
& s A_{\mu}^{a}=\frac{i}{g} D_{\mu}^{a b} c^{c}, \\
& s \zeta_{I}^{a}=i f^{a b c} \zeta_{I}^{b} c^{c}+c_{I}^{a}, \\
& s \bar{c}_{I}^{a}=-i f^{a b c} \bar{c}_{I}^{b} c^{c}-b_{I}^{a}, \\
& s \bar{c}^{a}=-b^{a}, \\
& s \mu^{2}=U^{2}, \\
& s \kappa=\mathcal{K}, \\
& s \lambda=\Lambda,
\end{aligned}
$$

$$
\begin{array}{r}
s c^{a}=-\frac{i}{g} f^{a b c} c^{b} c^{c}, \\
s c_{I}^{a}=-i f^{a b c} c_{I}^{b} c^{c}, \\
s b_{I}^{a}=i f^{a b c} b_{I}^{b} c^{c} \\
s b^{a}=0, \\
s U^{2}=0, \\
s \mathcal{K}=0, \\
s \Lambda=0 .
\end{array}
$$

The transformations of the gauge fixing parameters $\mu^{2}, \kappa, \lambda$ are introduced to ensure that all observables will be independent of them $[16,17]$.

\section{The vortex-free sector}

In Ref. [13] we have studied the renormalizability of this sector using the algebraic method. In this approach, the Ward Identities of the action are used to restrict the possible counter terms. In this regard, it is important to introduce external sources coupled with the non-linear BRST variations of the fields, i.e.

$$
\begin{aligned}
\Sigma_{\text {ext }}^{(1)}= & \int d^{4} x\left(\frac{i}{g} K_{\mu}^{a}\left(D_{\mu}^{a b} c^{b}\right)-\frac{1}{2} i \bar{C}^{a} f^{a b c} c^{b} c^{c}-i f^{a b c} \bar{L}_{I}^{a} c_{I}^{b} c^{c}+Q_{I}^{a}\left(i f^{a b c} \zeta_{I}^{b} c^{c}+c_{I}^{a}\right)\right. \\
& \left.+i f^{a b c} B_{I}^{a} b_{I}^{b} c^{c}-L_{I}^{a}\left(i f^{a b c} \bar{c}_{I}^{b} c^{c}+b_{I}^{a}\right)\right) .
\end{aligned}
$$


Moreover, an additional pair of external sources $\left(M_{I}^{a b}, N_{I}^{a b}\right)$ is introduced by means of the BRST exact term

$$
\Sigma_{e x t}^{(2)}=s \int d^{4} x M_{I}^{a b} \bar{c}^{a} \zeta_{I}^{b}
$$

They are introduced as a doublet, i.e. $s M_{I}^{a b}=N_{I}^{a b}, s N_{I}^{a b}=0$. To analyze the renormalizability, we employ the algebraic method [17]. In this regard, we write the quantum action $\Gamma$ at first order as

$$
\Gamma^{(1)}=S_{v f}+\hbar \Sigma_{C}^{v f}
$$

$\Sigma_{C}^{\mathrm{vf}}$ being the counter term, and impose on it all the Ward Identities satisfied by the tree level action $S_{\mathrm{vf}}$, given by

$$
S_{\mathrm{vf}}=S_{Y M}+\Sigma\left(S_{0}\right)+\Sigma_{\mathrm{ext}}^{(1)}+\Sigma_{\mathrm{ext}}^{(2)}
$$

\subsection{Ward Identities and renormalization}

Besides the Slavnov-Taylor, the action $S_{\text {vf }}$ satisfies 6 other Ward Identities. These are:

- The gauge-fixing equation,

$$
\frac{\delta S_{\mathrm{vf}}}{\delta b^{a}}=i f^{a b c} \eta_{I}^{b} \zeta_{I}^{c}-M_{I}^{a b} \zeta_{I}^{b}
$$

- The anti-ghost equation,

$$
\overline{\mathcal{G}}^{a} S_{\mathrm{vf}}=\left(\frac{\delta}{\delta \bar{c}^{a}}-i f^{a b c} \eta_{I}^{b} \frac{\delta}{\delta Q_{I}^{c}}+M_{I}^{a b} \frac{\delta}{\delta Q_{I}^{b}}\right) S_{\mathrm{vf}}=0 .
$$

- The ghost number equation,

$$
\begin{aligned}
\mathcal{N}_{\mathrm{gh}} S_{\mathrm{vf}} & =\int d^{4} x\left(c_{I}^{a} \frac{\delta}{\delta c_{I}^{a}}-\bar{c}_{I}^{a} \frac{\delta}{\delta \bar{c}_{I}^{a}}+c^{a} \frac{\delta}{\delta c^{a}}-\bar{c}^{a} \frac{\delta}{\delta \bar{c}^{a}}+\Lambda \frac{\delta}{\delta \Lambda}+\mathcal{K} \frac{\delta}{\delta \mathcal{K}}+\right. \\
& +U^{2} \frac{\delta}{\delta U^{2}}-K_{\mu}^{a} \frac{\delta}{\delta K_{\mu}^{a}}-Q_{I}^{a} \frac{\delta}{\delta Q_{I}^{a}}-B_{I}^{a} \frac{\delta}{\delta B_{I}^{a}}-2 \bar{C}^{a} \frac{\delta}{\delta \bar{C}^{a}}-2 \bar{L}_{I}^{a} \frac{\delta}{\delta \bar{L}_{I}^{a}} \\
& \left.+N_{I}^{a b} \frac{\delta}{\delta N_{I}^{a b}}-\xi_{I}^{a} \frac{\delta}{\delta \xi_{I}^{a}}+m_{I J}^{a b} \frac{\delta}{\delta m_{I J}^{a b}}\right) S_{\mathrm{vf}}=0
\end{aligned}
$$

- The global flavor symmetry,

$$
\begin{aligned}
Q S_{\mathrm{vf}} & =\left(\zeta_{I}^{a} \frac{\delta}{\delta \zeta_{I}^{a}}-b_{I}^{a} \frac{\delta}{\delta b_{I}^{a}}+c_{I}^{a} \frac{\delta}{\delta c_{I}^{a}}-\bar{c}_{I}^{a} \frac{\delta}{\delta \bar{c}_{I}^{a}}-\eta_{I}^{a} \frac{\delta}{\delta \eta_{I}^{a}}-Q_{I}^{a} \frac{\delta}{\delta Q_{I}^{a}}\right. \\
& +B_{I}^{a} \frac{\delta}{\delta B_{I}^{a}}+L_{I}^{a} \frac{\delta}{\delta L_{I}^{a}}-\bar{L}_{I}^{a} \frac{\delta}{\delta \bar{L}_{I}^{a}}-\kappa \frac{\delta}{\delta \kappa}-2 \lambda \frac{\delta}{\delta \lambda}-\mathcal{K} \frac{\delta}{\delta \mathcal{K}}-2 \Lambda \frac{\delta}{\delta \Lambda} \\
& \left.-N_{I}^{a b} \frac{\delta}{\delta N_{I}^{a b}}-M_{I}^{a b} \frac{\delta}{\delta M_{I}^{a b}}\right) S_{\mathrm{vf}}=0 .
\end{aligned}
$$


- The linearly broken rigid symmetry,

$$
\begin{aligned}
\mathcal{R} S_{\mathrm{vf}} & =\left(\zeta_{I}^{a} \frac{\delta}{\delta c_{I}^{a}}+\bar{c}_{I}^{a} \frac{\delta}{\delta b_{I}^{a}}-i f^{a b c} \eta_{I}^{a} \frac{\delta}{\delta N_{I}^{b c}}-B_{I}^{a} \frac{\delta}{\delta \bar{L}_{I}^{a}}+\bar{L}_{I}^{a} \frac{\delta}{\delta Q_{I}^{a}}-\kappa \frac{\delta}{\delta \mathcal{K}}-2 \lambda \frac{\delta}{\delta \Lambda}\right. \\
& \left.-M_{I}^{a b} \frac{\delta}{\delta N_{I}^{a b}}\right) S_{\mathrm{vf}}=\bar{L}_{I}^{a} c_{I}^{a}+L_{I}^{a} \bar{c}_{I}^{a}-\zeta_{I}^{a} Q_{I}^{a} .
\end{aligned}
$$

- The ghost equation,

$$
\begin{aligned}
\mathcal{G}^{a} S_{\mathrm{vf}} & =\left(\frac{\delta}{\delta c^{a}}+\left(f^{a b c} f^{c n m} \eta_{I}^{n}+i f^{a b n} M_{I}^{m n}\right) \frac{\delta}{\delta N_{I}^{m b}}\right) S_{\mathrm{vf}} \\
& =i f^{a b c}\left(\bar{C}^{b} c^{c}+Q_{I}^{b} \zeta_{I}^{c}+\bar{L}_{I}^{b} c_{I}^{c}+L_{I}^{b} \bar{c}_{I}^{c}+B_{I}^{b} b_{I}^{c}\right)+\frac{i}{g} D_{\mu}^{a b} K_{\mu}^{b}
\end{aligned}
$$

These symmetries restricted the possible counter terms dramatically. Indeed, we showed that the most general $\Sigma_{C}^{\mathrm{vf}}$ is given by

$$
\begin{aligned}
\Sigma_{C}^{\mathrm{vf}}= & \int d^{4} x\left(a_{0} S_{Y M}(A)+a_{1}\left(\partial_{\mu} b_{I}^{a} \partial_{\mu} \zeta_{I}^{a}+g f^{a b c} b_{I}^{a} \partial_{\mu} \zeta_{I}^{b} A_{\mu}^{c}+g f^{a b c} \partial_{\mu} b_{I}^{a} A_{\mu}^{b} \zeta_{I}^{c}+\right.\right. \\
& +g^{2} f^{a b e} f^{c d e} A_{\mu}^{a} b_{I}^{b} A_{\mu}^{c} \zeta_{I}^{d}+\partial_{\mu} \bar{c}_{I}^{a} \partial_{\mu} c_{I}^{a}+g f^{a b c} \bar{c}_{I}^{a}+g f^{a b c} \partial_{\mu} \bar{c}_{I}^{a} A_{\mu}^{b} c_{I}^{c} \partial_{\mu} c_{I}^{b} A_{\mu}^{c} \\
& \left.+g^{2} f^{a b e} f^{c d e} A_{\mu}^{a} \bar{c}_{I}^{b} A_{\mu}^{c} c_{I}^{d}\right)+a_{2} f^{I J K} f^{a b c}\left(\mathcal{K} \bar{c}_{I}^{a} \zeta_{J}^{b} \zeta_{K}^{c}-2 \kappa \bar{c}_{I}^{a} c_{J}^{b} \zeta_{K}^{c}-\kappa b_{I}^{a} \zeta_{J}^{b} \zeta_{K}^{c}\right)+ \\
& +a_{3, I J K L}^{a b c d}\left(\Lambda \bar{c}_{I}^{a} \zeta_{J}^{b} \zeta_{K}^{c} \zeta_{L}^{d}-3 \lambda \bar{c}_{I}^{a} c_{J}^{b} \zeta_{K}^{c} \zeta_{L}^{d}-\lambda b_{I}^{a} \zeta_{J}^{b} \zeta_{K}^{c} \zeta_{L}^{d}\right)+ \\
& \left.+a_{4}\left(U^{2} \bar{c}_{I}^{a} \zeta_{I}^{a}-\mu^{2} \bar{c}_{I}^{a} c_{I}^{a}-\mu^{2} b_{I}^{a} \zeta_{I}^{a}\right)\right)
\end{aligned}
$$

Then, we showed that it may be absorbed by a redefinition of the parameters, fields and sources of $S_{\mathrm{vf}}$, with appropriate renormalization factors.

\section{Sectors labeled by center vortices}

In Ref. [14], we considered a sector labeled by $n$ center vortices with guiding centers at the closed worldsurfaces $\Omega_{1}, \ldots, \Omega_{n}$. When they carry the same fundamental weight $\beta$, the associated defect may be written as $S_{0}=e^{i \chi \beta \cdot T}$, where $\chi$ is a multivalued angle with respect to $\Omega_{1}, \ldots, \Omega_{n}$. Here we are using the notation $\beta \cdot T=\left.\beta\right|_{q} T_{q}, T_{q}$ being the generators of the Cartan subalgebra of $s u(N)$. In these sectors, because of the gauge condition ..., the gaugefixed configurations of auxiliary fields will be of the form $\zeta_{I}=S_{0} q_{I} S_{0}^{-1}$, with $\left[q_{I}, T_{I}\right]=0$. This means that the components of $\zeta_{I}$ that rotate under $S_{0}$ must vanish at $\Omega=\Omega_{1} \cup \cdots \cup \Omega_{n}$. These are the components along the off-diagonal generators $T_{\alpha}, T_{\bar{\alpha}}$, with $\alpha \cdot \beta \neq 0, \alpha$ being the roots of $s u(N)$. To impose these boundary conditions, we followed the method of Ref. [18], where the delta function that implements the boundary conditions is exponentiated as follows

$$
\begin{aligned}
& \prod_{\gamma} \delta_{\Omega}\left(\zeta_{I}^{\gamma}\right) \delta_{\Omega}\left(\zeta_{I}^{\bar{\gamma}}\right)= \\
& \int[D \lambda] e^{i \sum_{\gamma} \int d \sigma_{1} d \sigma_{2} \sqrt{g\left(\sigma_{1}, \sigma_{2}\right)}\left(\lambda_{I}^{\gamma}\left(\sigma_{1}, \sigma_{2}\right) \zeta_{I}^{\gamma}\left(x\left(\sigma_{1}, \sigma_{2}\right)\right)+\lambda_{I}^{\bar{\gamma}}\left(\sigma_{1}, \sigma_{2}\right) \zeta_{I}^{\bar{\gamma}}\left(x\left(\sigma_{1}, \sigma_{2}\right)\right)\right)}
\end{aligned}
$$


Moreover, in order to account for the BRST invariance of these boundary conditions, we should also impose $s \zeta_{I}^{\gamma}=s \zeta_{I}^{\bar{\gamma}}=0$ on $\Omega$ [19]. To this purpose, we represented this expression in an alternative, more symmetric form, by means of the Symanzik method [20], where a generic Lie algebra valued source $J(x)$ was introduced. Then

$$
\prod_{\gamma} \delta_{\Omega}\left(\zeta_{I}^{\gamma}\right) \delta_{\Omega}\left(\zeta_{I}^{\bar{\gamma}}\right) \delta_{\Omega}\left(s \zeta_{I}^{\gamma}\right) \delta_{\Omega}\left(s \zeta_{I}^{\bar{\gamma}}\right) \rightarrow \int[D \lambda][D \xi] e^{-\Sigma^{\mathrm{b} . c .}(\lambda, \xi, J)}
$$

with the additional fields satisfying

$$
\begin{array}{ll}
s \xi_{I}^{a}=\lambda_{I}^{a}, & s \lambda_{I}^{a}=0 \\
s J=0 . &
\end{array}
$$

The expression of interest is recovered when $J$ is set to take its physical values (see Ref. [14]), and automatically takes into account the BRST invariance of the boundary conditions. Then, it is convenient to introduce external sources $m_{I J}^{a b}, n_{I J}^{a b}$ in order to preserve the symmetries of the vortex-free sector. In order to guarantee that these do not alter the physical theory, they are introduced as a BRST doublet, i.e.

$$
s m_{I J}^{a b}=-n_{I J}^{a b}, \quad s n_{I J}^{a b}=0 .
$$

Therefore, the full action in these sectors is given by

$$
\Sigma^{\text {vortex }}=\Sigma\left(S_{0}\right)+S_{\mathrm{ext}}^{(1)}+\Sigma_{\mathrm{ext}}^{(2)}+\Sigma_{\mathrm{ext}}^{(3)}+\Sigma^{\text {b.c. }} .
$$

To analyze the renormalizability of $\Sigma^{\text {vortex }}$, we proceeded similarly as in the vortex-free case. Namely, we imposed the Ward Identities in the quantum action to restrict the possible counterterms, and studied the stability of the action.

\subsection{Ward Identities and renormalization}

Three new Ward Identities are satisfied in the center vortex sectors. These involve the new fields $\lambda_{I}, \xi_{I}, J, m_{I J}, n_{I J}$, and are given by

- The $J$ equation,

$$
\mathcal{J}^{a} \Sigma^{\mathrm{vortex}}=\left(\frac{\delta}{\delta J^{a}}-f^{a b c} \delta_{I J} \frac{\delta}{\delta m_{I J}^{b c}}\right) \Sigma^{\mathrm{vortex}}=0 .
$$

- Global symmetry in the boundary-conditions sector,

$$
\begin{aligned}
\mathcal{F} \Sigma^{\text {vortex }} & =\left(\xi_{I}^{a} \frac{\delta}{\delta \xi_{I}^{a}}+\lambda_{I}^{a} \frac{\delta}{\delta \lambda_{I}^{a}}-J^{a} \frac{\delta}{\delta J^{a}}-n_{I J}^{a b} \frac{\delta}{\delta n_{I J}^{a b}}-m_{I J}^{a b} \frac{\delta}{\delta m_{I J}^{a b}}\right) \Sigma^{\mathrm{vortex}} \\
& =0 .
\end{aligned}
$$

- The linearly broken $\lambda$ equation,

$$
\Lambda_{I}^{a} \Sigma^{\mathrm{vortex}}=\frac{\delta \Sigma^{\mathrm{vortex}}}{\delta \lambda_{I}^{a}}=f^{a b c} \zeta_{I}^{b} J^{c} .
$$

In Ref. [14], we showed that $\Sigma_{C}^{\mathrm{vortex}}=\Sigma_{C}^{\mathrm{vf}}$, and that the action is stable in the vortex sectors. 


\section{Discussion}

Recently, a new way to quantize Yang-Mills theory based on a local gauge fixing procedure, so as to avoid the presence of Gribov copies, was proposed. The path integral was written as a sum over inequivalent sectors labeled by center vortices, and the gauge is fixed with a sector dependent gauge condition. Within this procedure, observables of Yang-Mills theory are evaluated as a sum over partial contributions of each possible center vortex sector, providing a glimpse of a path from Yang-Mills theory to phenomenological center-vortex ensembles. In Ref. [13], we established the renormalizability of the vortex-free sector by using the algebraic method, and then, in Ref. [14], this was generalized to a sector containing an arbitrary number of elementary center vortices. The BRST structure guaranteed the independence of observables from the gauge fixing parameters, and the introduction of appropriate external sources allowed us to to establish a rich set of Ward Identities. These restrictive symmetries implied that the counter-term is the same in all the analyzed sectors, and so is the number of independent renormalization parameters. These results show that this first principles YangMills ensemble is indeed calculable.

\section{Acknowledgments}

The Coordenação de Aperfeiçoamento de Pessoal de Nível Superior - Brasil (CAPES) Finance Code 001, the Deutscher Akademischer Austauschdienst (DAAD) and the Conselho Nacional de Desenvolvimento Científico e Tecnológico (CNPQ) are acknowledged for the financial support.

\section{References}

[1] I. M. Singer, Commun. Math. Phys. 60 (1978) 7-12.

[2] V. N. Gribov, Nucl. Phys. B139 (1978) 1.

[3] D. Zwanziger, Nucl. Phys. B323 (1989) 513-544.

[4] D. Zwanziger, Nucl. Phys. B399 (1993) 477-513.

[5] D. Dudal, R. F. Sobreiro, S. P. Sorella, and H. Verschelde, Phys. Rev. D72 (2005) 014016.

[6] D. Dudal, J. A. Gracey, S. P. Sorella, N. Vandersickel, and H. Verschelde, Phys. Rev. D78 (2008) 065047.

[7] D. Dudal, S. P. Sorella, and N. Vandersickel, Phys. Rev. D84 (2011) 065039.

[8] M. A. L. Capri, D. Dudal, D. Fiorentini, M. S. Guimaraes, I. F. Justo, A. D. Pereira, B. W. Mintz, L. F. Palhares, R. F. Sobreiro, and S. P. Sorella, Phys. Rev. D 92 no. 4, (2015) 045039.

[9] M. A. L. Capri, D. Dudal, D. Fiorentini, M. S. Guimaraes, I. F. Justo, A. D. Pereira, B. W. Mintz, L. F. Palhares, R. F. Sobreiro, and S. P. Sorella, Phys. Rev. D94 no. 2, (2016) 025035.

[10] L. E. Oxman and G. C. Santos-Rosa, Phys. Rev. D 92 (2015) 125025.

[11] Greensite, J. An Introduction to the Confinement Problem, 2nd ed.; Springer Nature Switzerland: Cham, Switzerland, 2020.

[12] D. R. Junior, L. E. Oxman, G. M. Simões, Universe 7(8), (2021) 253

[13] D. Fiorentini, D. R. Junior, L. E. Oxman, and R. F. Sobreiro, Phys. Rev. D 101 no. 8, (2020) 085007.

[14] D. Fiorentini, D. R. Junior, L. E. Oxman, R. F. Sobreiro, arXiv:2108.11361 
[15] D. Fiorentini, D. R. Junior, L. E. Oxman, G. M. Simões, and R. F. Sobreiro, Phys. Rev. D 103 (2021) 114010.

[16] O. Piguet and K. S., Nucl. Phys. B253 (1985) 517-540.

[17] O. Piguet and S. P. Sorella, "Algebraic renormalization: Perturbative renormalization, symmetries and anomalies". Lect. Notes Phys. Monogr. 28 (1995) 1-134.

[18] R. Golestanian and M. Kardar, Phys. Rev. A 58 (1998) 1713-1722.

[19] I. G. Moss and P. J. Silva,Phys. Rev. D 55 (1997) 1072-1078.

[20] K. Symanzik, Commun. Math. Phys. 16 (1970) 48-80. 